Rapid Reviews COVID-19

\title{
Reviews of "Wastewater Surveillance of SARS-CoV- 2 across 40 U.S. states"
}

\author{
Rosa Pintó ${ }^{1}$, Ahmed Yousef ${ }^{2}$, Glenn Simmons ${ }^{3}$ \\ ${ }^{1}$ Full Professor, University of Barcelona: Universitat de Barcelona, Genetics, Microbiology and \\ Statistics, Spain, \\ ${ }^{2}$ Department of Chemistry, Khalifa University of Science and Technology, Abu Dhabi, United Arab \\ Emirates, \\ ${ }^{3}$ Assistant Professor, University of Minnesota, Biomedical Sciences
}

Published on: Jun 16, 2021

License: Creative Commons Attribution 4.0 International License (CC-BY 4.0). 
To read the original manuscript, click the link above.

Summary of Reviews: This preprint reports a high detection rate of SARS-CoV-2 using wastewater surveillance and finds that virus detection precedes clinical indicators. Reviewers deem these findings potentially informative, recommending large scale studies to confirm findings.

Reviewer 1 (Rosa Pintó)

Reviewer 2 (Ahmed Yousef) | प्र००

\title{
Reviewer 3 (Glenn Simmons) |
}

\author{
RR:C19 Strength of Evidence Scale Key. \\ प्रमप्र = Misleading \\ प्र $\square \square \square=$ Not Informative \\ ㄷํ $\square \square=$ Potentially Informative \\ प्राप्र = Reliable \\ $\operatorname{l|c|c|c|}=$ Strong
}

To read the reviews, click the links below. 